\title{
A quadrature voltage-controlled oscillator using phase-adjusting architecture for suppressing phase noise
}

\author{
Mamoru Ugajin ${ }^{1, \text { a) }}$, Yuya Kakei ${ }^{1}$, and Nobuyuki Itoh ${ }^{2}$
}

Abstract A low-phase-noise 25-GHz quadrature voltage-controlled oscillator (VCO) using 180-nm TSMC CMOS is presented. To equalize the oscillation frequency to the LC-resonant frequency, a phase shifter at a transistor gate adjusts a phase delay due to an RC delay in a transistor. This phase-adjusting architecture extends operation bias range and suppresses phase noise by 6 to $9 \mathrm{~dB}$ compared with a conventional differential VCO. Keywords: phase noise, voltage-controlled oscillator, gate delay, phaseadjusting architecture

Classification: Integrated circuits (memory, logic, analog, RF, sensor)

\section{Introduction}

High-bit-rate wireless communications demand high carrier frequencies [1], such as 24-GHz WLAN [2], IEEE802.11 ad [3], and 24-29 GHz 5G mobile phones [4]. One of essential circuit blocks in the high-frequency wireless systems is a voltage-controlled oscillator (VCO). Various quadrature (4-phase) VCOs are investigated [5, 6, 7, 8, 9] for quadrature signal processing $[10,11,12,13,14,15,16,17]$ in the high-performance wireless systems, however, a highfrequency VCO usually needs a very fine CMOS technology and/or a special high-Q inductor process $[18,19,20,21,22$, 23 ] for suppressing the phase noise. Thus, their process cost could be considerably high. In this letter, a quadrature VCO using a phase-adjusting architecture for suppressing phase noise is presented. This architecture can be implemented without process-cost increase.

\section{Concept of phase adjusting and circuit design}

Figure 1 shows the circuit structure and the ac-analysis results of a differential LC VCO. The open-loop gain and phase were calculated with a dummy LC amplifier for adding gate capacitances to an LC parallel resonant circuit. The gain peak locates at the LC resonant frequency of $29.4 \mathrm{GHz}$, however, the oscillation occurs at $28.7 \mathrm{GHz}$ because of oscillation condition $\left(180^{\circ}\right.$ phase delay). The difference between the LC resonant frequency and the oscillation frequency arises from an RC delay in a transistor. The RC delay causes

\footnotetext{
${ }^{1}$ Nippon Institute of Technology, Minami-Saitama, Saitama 345-8501, Japan

2 Okayama Prefectural University, Soja-shi, Okayama 719-1197, Japan

a) uga@ nit.ac.jp
}

DOI: 10.1587/elex.18.20210173

Received April 15, 2021

Accepted April 21, 2021

Publicized April 28, 2021

Copyedited May 25, 2021

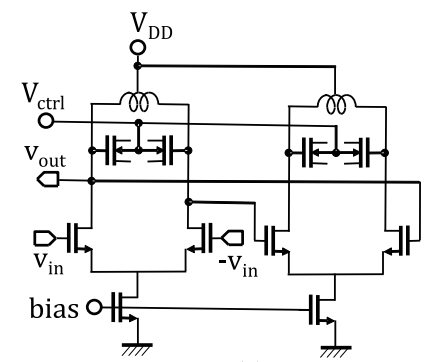

(a)

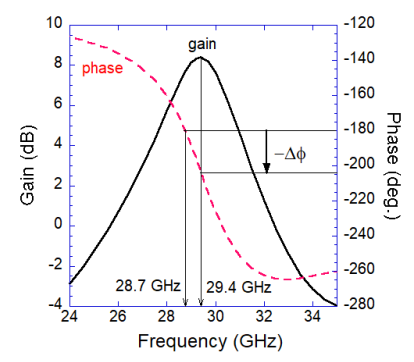

(b)
Fig. 1 (a) The circuit structure and (b) the gain and phase results of open-loop ac analysis for a differential LC VCO.

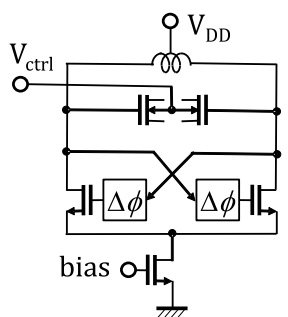

(a)

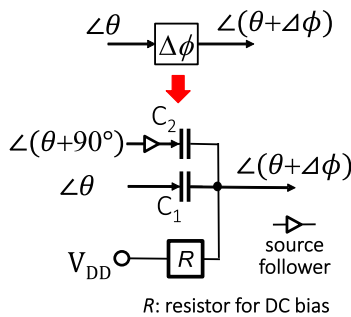

(b)
Fig. 2 (a) A differential VCO with two phase shifters and (b) a phase sifter circuit using a weight-average architecture with two capacitors and a source follower circuit. $R$ is a resistor for DC bias.

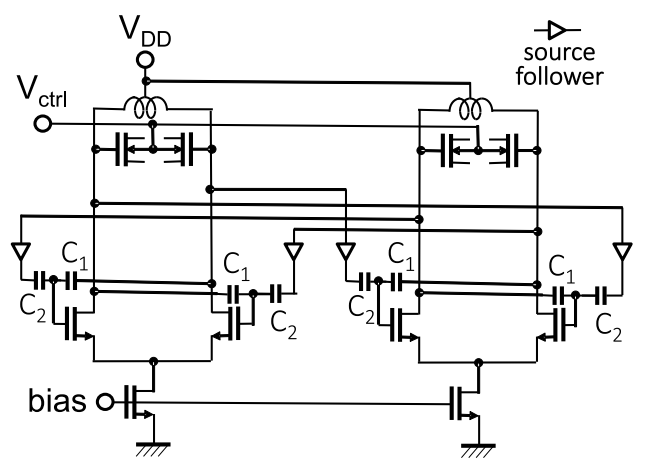

Fig. 3 Circuit architecture of a quadrature VCO using four phase adjusts formed by 8 capacitors and 4 source followers.

phase delay $\left(-\triangle \phi \approx-24^{\circ}\right)$ and lowers the oscillation frequency.

The loop gain of VCO should be maximum to suppress its phase noise [24], thus the oscillation frequency should be the LC resonant frequency. Therefore $\Delta \phi$-phase shifters at two gate inputs of a differential VCO (depicted in Fig. 2(a)) are thought to be very helpful to adjust phase delay and suppress phase noise. The $\Delta \phi$-phase shifter was designed 
using a weight-average architecture with two capacitors and a source follower circuit as shown in Fig. 2 (b).

Figure 3 shows the quadrature VCO circuit using the phase adjusting architecture. Four $\Delta \phi$-phase shifters are added to two conventional differential VCOs.

\section{Measurement results and discussion}

Two conventional differential VCOs [25, 26, 27, 28, 29] and the phase-adjusting quadrature $\mathrm{VCO}$ were fabricated using TSMC 180-nm CMOS technology (as shown in Fig. 4). The conventional VCOs were realized by just removing the $\Delta \phi$ phase shifters from the phase-adjusting quadrature VCO. The values of $C_{1}$ and $C_{2}$ of the $\Delta \phi$-phase shifter were 600 $\mathrm{fF}$ and $60 \mathrm{fF}$, respectively.

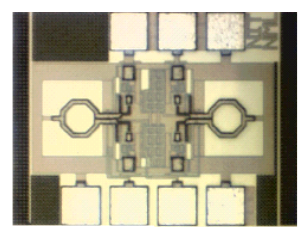

(a)

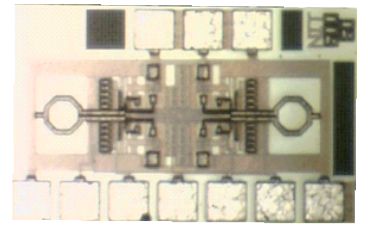

(b)
Fig. 4 Chip microphotographs of (a) two conventional differential VCOs and (b) a phase-adjusting quadrature VCO.

The phase noises of the VCOs were measured with various bias voltages, $V_{D D}=2.2 \mathrm{~V}$ and $V_{c t r l}=0 \mathrm{~V}$. Figure 5 shows the relation between phase noise at $1-\mathrm{MHz}$ offset frequency and current consumption. The current consumption shows the current of the one conventional differential VCO and that of the phase-adjusting quadrature VCO with four source followers. In terms of bias setting, the quadrature VCO has a larger operation range than that of a conventional VCO. Oscillation frequencies were $28.05 \mathrm{GHz}$ for the conventional $\mathrm{VCO}$ and $25.17 \mathrm{GHz}$ for the quadrature VCO. The $\Delta \phi$ phase shifter worked not only as a phase shifter but also as an additional capacitor and thus both of the LC-resonant frequency and the oscillation frequency of the phase-adjusting quadrature VCO were lowered. The ranges of oscillation frequencies were 28.05 to $28.73 \mathrm{GHz}$ for the conventional $\mathrm{VCO}$ and 25.17 to $25.73 \mathrm{GHz}$ for the quadrature $\mathrm{VCO}$ when $V_{c t r l}$ was changed from 0 to $2.2 \mathrm{~V}$. The additional capacitance of the $\triangle \phi$-phase shifter reduced the frequency range by about $10 \%$. The figure-of-merit (FOM) is defined as [30]:

$$
F O M=L(\triangle f)-20 \log \left(\frac{f_{0}}{\triangle f}\right)+10 \log \left(\frac{P_{D C}}{1 m W}\right) .
$$

The best FOMs of the VCOs in Fig. 5 are evaluated as $-175.1 \mathrm{dBc} / \mathrm{Hz}$ and $-176.4 \mathrm{dBc} / \mathrm{Hz}$ for the conventional $\mathrm{VCO}$ and the phase-adjusting quadrature VCO, respectively. A quadrature VCO usually consumes two times larger current than that of a differential VCO, thus the FOM improvement is effectively $4.3 \mathrm{~dB}$. Figure 6 shows the offsetfrequency dependence of phase noise at the conditions of minimum phase noise in Fig. 5. Thus in Fig. 6, the current consumptions of the conventional VCOs and the phaseadjusting quadrature VCO were $15.4 \mathrm{~mA}$ and $41.2 \mathrm{~mA}$, respectively. The phase-adjusting architecture suppresses phase noise about $9 \mathrm{~dB}$ and $6 \mathrm{~dB}$ at $100-\mathrm{kHz}$ and $1-\mathrm{MHz}$ offset frequencies, respectively. The 3-dB reduction of phasenoise suppression at $1-\mathrm{MHz}$ offset frequency was probably due to measurement errors as shown in Fig. 6.

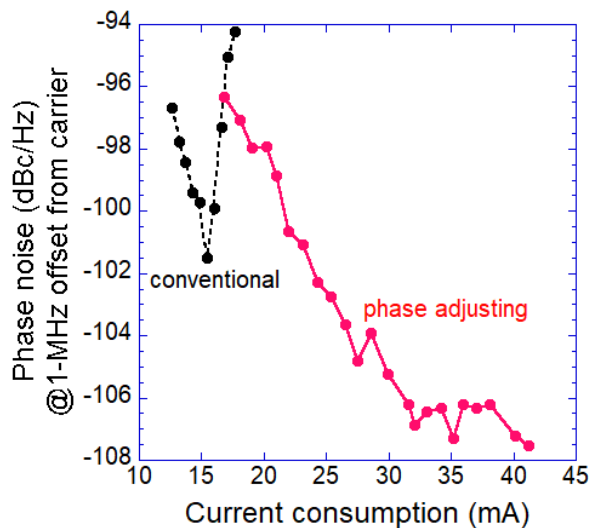

Fig. 5 Relation between phase noise and current consumption. Phase noise was measured at $1-\mathrm{MHz}$ offset frequency from the carrier frequency.

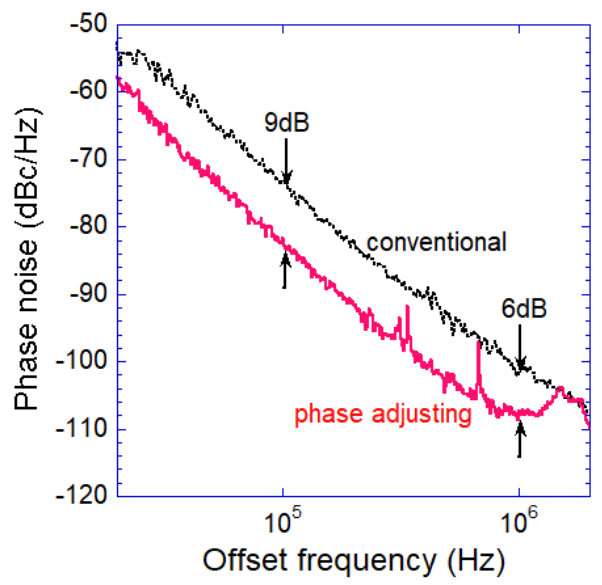

Fig. 6 Measured phase noise as a function of the offset frequency from the carrier frequency.

Performance comparison with recently published CMOS LC VCOs is illustrated in Table I [ $31,32,33,34]$. All VCOs are quadrature VCOs. The proposed VCO shows phasenoise performances comparable to the finer-process VCOs.

Table I Performance comparison

\begin{tabular}{|c|c|c|c|c|c|}
\hline Ref & $\begin{array}{c}\text { This } \\
\text { work }\end{array}$ & {$[31]$} & {$[32]$} & {$[33]$} & {$[34]$} \\
\hline \hline process (nm) & 180 & 180 & 130 & 65 & 65 \\
\hline$f_{\text {osc }}(\mathrm{GHz})$ & 25.17 & 19.85 & 20.17 & 20.88 & 26 \\
\hline T.R. (\%) & 2.2 & 10.4 & - & 17.9 & 14.4 \\
\hline$V_{D D}(\mathrm{~V})$ & 2.2 & 1.8 & 1.2 & 1.2 & - \\
\hline$P_{D C}(\mathrm{~mW})$ & 90.6 & 40.3 & 28.8 & 80.0 & 11.8 \\
\hline $\begin{array}{c}\text { PN @ } 1 \mathrm{MHz} \\
(\mathrm{dBc} / \mathrm{Hz})\end{array}$ & -107.5 & -111.5 & -102.4 & -100 & -100 \\
\hline FOM (dBc/Hz) & -176.4 & -181.4 & -173.9 & -173.4 & -177.6 \\
\hline
\end{tabular}

\section{Conclusion}

A low-phase-noise $25-\mathrm{GHz}$ quadrature VCO using $180-\mathrm{nm}$ TSMC CMOS is presented. A phase shifter equalizes the 
oscillation frequency to the LC-resonant frequency, extends operation bias range and suppresses phase noise by 6 to 9 $\mathrm{dB}$ compared to a conventional differential VCO.

\section{Acknowledgments}

This work was supported through the activities of VDEC, The University of Tokyo, in collaboration with Cadence Design Systems, Inc.; and JSPS KAKENHI Grant Number $18 \mathrm{~K} 04288$.

\section{References}

[1] C.E. Shannon: "A mathematical theory of communication," The Bell System Technical Journal 27 (1948) 379 (DOI: 10.1002/j.1538-7305. 1948.tb01338.x).

[2] A. Mazzanti, et al.: "A $24 \mathrm{GHz}$ sub-harmonic receiver front-end with integrated multi-phase LO generation in 65nm CMOS," ISSCC Dig. Tech. Papers (2008) 216 (DOI: 10.1109/ISSCC.2008.4523134).

[3] K. Okada, et al.: "Full four-channel 6.3-Gb/s 60-GHzCMOS transceiver with low-power analog and digital baseband circuitry," IEEE J. Solid-State Circuits 48 (2013) 46 (DOI: 10.1109/JSSC.2012. 2218066).

[4] S. Ek, et al.: "A 16-20 GHz LO system with 115 fs jitter for 24-30 GHz 5G in 28 nm FD-SOI CMOS," 43rd IEEE European Solid State Circuits Conference (2017) 110 (DOI: 10.1109/ESSCIRC.2017. 8094573).

[5] N. Xi, et al:: "A low phase noise, high phase accuracy quadrature LC-VCO with dual-tail current biasing to insert reconfigurable phase delay,” IEEE Trans. Circuits Syst. II, Exp. Brief 67 (2020) 19409349 (DOI: 10.1109/TCSII.2019.2923235).

[6] V. Natarajan, et al.: "Low noise RF quadrature VCO using tail-switch network-based coupling in $40 \mathrm{~nm}$ CMOS," IEEE Custom Integrated Circuits Conference (2018) 17753998 (DOI: 10.1109/CICC.2018. 8357040).

[7] Q. Xia, et al.: "A low phase noise X-band quadrature VCO by using transconductance linearization technique," International Conference on Microwave and Millimeter Wave Technology (2019) 19357510 (DOI: 10.1109/ICMMT45702.2019.8992075).

[8] W.-C. Lai, et al.: "Quadrature VCO via transformer-coupled transmission line," International Workshop on the Electromagnetic Compatibility of Integrated Circuits (2019) 19222482 (DOI: 10.1109/ EMCCompo.2019.8919938).

[9] V. Aggarwal and S. Aniruddhan: "A 27-29GHz integer-N pLL with quadrature phases for 5G applications," IEEE MTT-S International Microwave Conference on Hardware and Systems for 5G and Beyond (2019) 19873322 (DOI: 10.1109/IMC-5G47857.2019.9160374).

[10] M. Ugajin, et al.: "A $1 \mathrm{~V} 12 \mathrm{~mW} 2 \mathrm{GHz}$ receiver with $49 \mathrm{~dB}$ image rejection in CMOS/SIMOX," ISSCC Dig. Tech. Papers (2001) 6924578 (DOI: 10.1109/ISSCC.2001.912641).

[11] M. Ugajin, et al.: "A 1-V 2-GHz RF receiver with $49 \mathrm{~dB}$ of image rejection in CMOS/SIMOX," IEICE Trans. Fundamentals E85-A (2002) 293

[12] M. Ugajin, et al.: "A 1-V CMOS/SOI bluetooth RF transceiver for compact mobile applications," Symposium on VLSI Circuits. Digest of Technical Papers (2003) 7869128 (DOI: 10.1109/VLSIC.2003. 1221179).

[13] M. Ugajin and T. Tsukahara: "A 1-V 2.4-GHz FSK receiver with a complex BPF and a frequency doubler in CMOS/SOI," Proceedings of the IEEE 2003 Custom Integrated Circuits Conference (2003) 7838840 (DOI: 10.1109/CICC.2003.1249380).

[14] M. Ugajin, et al:: "A 1-V 2.4-GHz downconverter for FSK wireless applications with a complex BPF and a frequency doubler in CMOS/SOI," IEICE Trans. Electron. E87-C (2004) 888.

[15] M. Ugajin, et al.: "High-image-rejection wireless-receiver architecture with a 3-phase active RC complex filter," IEICE Electron. Express 12 (2015) 20150329 (DOI: 10.1587/elex.12.20150329).

[16] M. Ugajin and T. Shindou: "Image-rejection-performance assessment of double-conversion wireless receiver with 3-phase complex filter using montecarlo simulation," International Symposium on Communications and Information Technologies (2015) 299 (DOI: 10.1109/ISCIT.2015.7458366).

[17] M. Ugajin, et al.: "An $\left(N+N^{2}\right)$-mixer architecture for a high-imagerejection wireless receiver with an $N$-phase active complex filter," IEICE Trans. Fundamentals E100-A (2017) 1008 (DOI: 10.1587/ transfun.E100.A.1008).

[18] M.K. Yapici, et al.: "Post-CMOS on-chip integration of 3D MEMS inductors using a novel chip embedding technique," The 17th International Conference on Solid-State Sensors, Actuators and Microsystems (2013) 13838263 (DOI: 10.1109/Transducers.2013.6626718).

[19] W. Huang, et al.: "A CMOS-compatible on-chip self-rolled-up inductors for RF/mm-wave applications," IEEE MTT-S International Microwave Symposium (2017) 17238782 (DOI: 10.1109/MWSYM. 2017.8058953)

[20] X. Li, et al.: "On-chip inductor for millimeter-wave regime," IEEE International Symposium on Radio-Frequency Integration Technology (2019) 19224687 (DOI: 10.1109/RFIT.2019.8929118).

[21] K. Okada, et al:: "On-chip high- $Q$ variable inductor using waferlevel chip-scale package technology," IEEE Trans. Electron Devices 53 (2006) 9066871 (DOI: 10.1109/TED.2006.880815)

[22] K. Itoi, et al.: "On-chip high-Q spiral $\mathrm{Cu}$ inductors embedded in wafer-level chip-scale package for silicon RF application," IEEE MTT-S International Microwave Symposium Digest (2004) 8095743 (DOI: 10.1109/MWSYM.2004.1335843).

[23] H. Namba, et al:: "On-chip vertically coiled solenoid inductors and transformers for RF SoC using 90nm CMOS interconnect technology,' IEEE Radio Frequency Integrated Circuits Symposium (2011) 12094918 (DOI: 10.1109/RFIC.2011.5940640).

[24] T.H. Lee and A. Hajimiri: "Oscillator phase noise: a tutorial," IEEE J. Solid-State Circuits 35 (2000) 326 (DOI: 10.1109/4.826814)

[25] Y. Sakamoto, et al.: "Phase noise characteristics of VCOs utilizing various structural 3D-striped inductor," Proceedings of 2018 AsiaPacific Microwave Conference (2018) 974 (DOI: 10.23919/APMC 2018.8617495).

[26] T. Yuki, et al.: "A study of $24 \mathrm{GHz} 3 \mathrm{D}$-striped inductor VCO with gradually changed metal width of inductor," IEEE International Symposium on Radio-Frequency Integration Technology (2020) 20064245 (DOI: 10.1109/RFIT49453.2020.9226227).

[27] Y. Sakamoto, et al.: " $24 \mathrm{GHz}$ low-phase-noise VCO using 3D-striped inductor utilized thin-metal layers," IEEE Asia Pacific Microwave Conference (APMC) 17486408 (DOI: 10.1109/APMC.2017. 8251682).

[28] N. Itoh, et al.: "Striped inductor for quasi millimeter wave voltagecontrolled oscillator," Proceedings of 2013 Asia-Pacific Microwave Conference (2013) 319 (DOI: 10.1109/APMC.2013.6695132).

[29] N. Itoh, et al.: "A study of striped inductor for K- and Ka-band voltage-controlled oscillators," IEICE Trans. Electron E99-C (2016) 614 (DOI: 10.1587/transele.E99.C.614).

[30] B. Razavi: RF Microelectronics (Prentice Hall 2013) 2nd ed. 570.

[31] S. Ko, et al.: "20 GHz integrated CMOS frequency sources with a quadrature VCO using transformers," IEEE Radio Frequency Integrated Circuits Symposium (2004) 8008808 (DOI: 10.1109/RFIC. 2004.1320593).

[32] M. Hossain and A.C. Carusone: " $20 \mathrm{GHz}$ low power QVCO and deskew techniques in $0.13 \mu \mathrm{m}$ digital CMOS," IEEE Custom Integrated Circuits Conference (2008) 10388838 (DOI: 10.1109/CICC.2008. 4672117).

[33] O. Richard, et al.: "A 17.5-to-20.94GHz and 35-to-41.88GHz PLL in $65 \mathrm{~nm}$ CMOS for wireless HD applications," IEEE International Solid-State Circuits Conference (2010) 11205053 (DOI: 10.1109/ ISSCC.2010.5433941)

[34] J.-C. Chien, et al:: "A 26-GHz low-phase-error in-phase-coupled QVCO using modified bi-directional diodes," IEEE Radio Frequency Integrated Circuits Symposium (2014) 14447179 (DOI: 10.1109/ RFIC.2014.6851712). 